\title{
JCOG0911 INTEGRA study: a randomized screening phase II trial of interferon $\beta$ plus temozolomide in comparison with temozolomide alone for newly diagnosed glioblastoma
}

\author{
Toshihiko Wakabayashi ${ }^{1} \cdot$ Atsushi Natsume $^{1}$ (1) Junki Mizusawa ${ }^{2} \cdot$ Hiroshi Katayama $^{2} \cdot$ Haruhiko Fukuda $^{2}$. \\ Minako Sumi ${ }^{3} \cdot$ Ryo Nishikawa $^{4} \cdot$ Yoshitaka Narita $^{5} \cdot$ Yoshihiro Muragaki $^{6} \cdot$ Takashi Maruyama $^{6}$. Tamio Ito $^{7}$. \\ Takaaki Beppu $^{8}$. Hideo Nakamura ${ }^{9}$. Takamasa Kayama ${ }^{10}$. Shinya Sato ${ }^{10} \cdot$ Motoo Nagane $^{11} \cdot$ Kazuhiko Mishima $^{4}$. \\ Yoko Nakasu $^{12} \cdot$ Kaoru Kurisu $^{13} \cdot$ Fumiyuki Yamasaki $^{13} \cdot$ Kazuhiko Sugiyama $^{14} \cdot$ Takanori Onishi $^{15} \cdot$ Yasuo Iwadate $^{16}$. \\ Mizuhiko Terasaki ${ }^{17}$. Hiroyuki Kobayashi ${ }^{18} \cdot$ Akira Matsumura $^{19} \cdot$ Eiichi Ishikawa $^{19} \cdot$ Hikaru Sasaki $^{20}$. \\ Akitake Mukasa ${ }^{21}$. Takayuki Matsuo ${ }^{22}$. Hirofumi Hirano ${ }^{23} \cdot$ Toshihiro Kumabe $^{24,25}$. Nobusada Shinoura ${ }^{26}$. \\ Naoya Hashimoto ${ }^{27}$. Tomokazu Aoki ${ }^{28}$. Akio Asai $^{29} \cdot$ Tatsuya Abe $^{30}$. Atsuo Yoshino ${ }^{31}$. Yoshiki Arakawa ${ }^{32}$. \\ Kenichiro Asano ${ }^{33} \cdot$ Koji Yoshimoto $^{34}$. Soichiro Shibui ${ }^{5}$. Members of Japan Clinical Oncology Group Brain Tumor \\ Study Group (JCOG-BTSG)
}

Received: 13 November 2017 / Accepted: 14 February 2018 / Published online: 20 March 2018 (c) The Author(s) 2018

\begin{abstract}
Purpose This study explored the superiority of temozolomide (TMZ) + interferon $\beta$ (IFN $\beta$ ) to standard TMZ as treatment for newly diagnosed glioblastoma (GBM) via randomized phase II screening design.

Experimental design Eligibility criteria included histologically proven GBM, with 50\% of the tumor located in supratentorial areas, without involvement of the optic, olfactory nerves, and pituitary gland and without multiple lesions and dissemination. Patients in the TMZ + radiotherapy (RT) arm received RT (2.0 Gy/fr/day, $30 \mathrm{fr}$ ) with TMZ ( $75 \mathrm{mg} / \mathrm{m}^{2}$, daily) followed by TMZ maintenance $\left(100-200 \mathrm{mg} / \mathrm{m}^{2} /\right.$ day, days $1-5$, every 4 weeks) for 2 years. Patients in the TMZ + IFN $\beta+R T$ arm intravenously received IFN $\beta$ ( 3 MU/body, alternative days during RT and day 1, every 4 weeks during maintenance period) and $\mathrm{TMZ}+\mathrm{RT}$. The primary endpoint was overall survival (OS). The planned sample size was 120 (one-sided alpha 0.2 ; power 0.8).

Results Between Apr 2010 and Jan 2012, 122 patients were randomized. The median OS with TMZ + RT and $\mathrm{TMZ}+\mathrm{IFN} \beta+\mathrm{RT}$ was 20.3 and 24.0 months (HR 1.00 , 95\% CI $0.65-1.55$; one-sided log rank $\mathrm{P}=0.51$ ). The median progression-free survival times were 10.1 and 8.5 months (HR 1.25, 95\% CI 0.85-1.84). The incidence of neutropenia with the $\mathrm{TMZ}+\mathrm{RT}$ and the TMZ + IFN $\beta+\mathrm{RT}$ (grade 3-4, CTCAE version 3.0) was 12.7 versus $20.7 \%$ during concomitant period and was 3.6 versus $9.3 \%$ during maintenance period. The incidence of lymphopenia was 54.0 versus $63.8 \%$ and 34.5 versus $41.9 \%$. Conclusions $\mathrm{TMZ}+\mathrm{IFN} \beta+\mathrm{RT}$ is not considered as a candidate for the following phase III trial, and TMZ + RT remained to be a most promising treatment. This trial was registered with the UMIN Clinical Trials Registry: UMIN000003466.
\end{abstract}

Keywords Glioblastoma $\cdot$ Interferon-beta $\cdot$ Temozolomide $\cdot$ MGMT $\cdot$ RCT

\begin{tabular}{|c|c|c|c|}
\hline \multicolumn{2}{|c|}{ Abbreviations } & \multirow{2}{*}{$\begin{array}{l}\text { MU } \\
\text { GBM }\end{array}$} & \multirow{2}{*}{$\begin{array}{l}\text { Million unit } \\
\text { Glioblastoma }\end{array}$} \\
\hline TMZ & Temozolomide & & \\
\hline $\operatorname{IFN} \beta$ & Interferon $\beta$ & MGMT & $O^{6}$-methylguanine-DNA methyltransferase \\
\hline RT & Radiotherapy & PTV & Planning target volume \\
\hline \multirow[t]{3}{*}{ OS } & Overall survival & GTV & Gross tumor volume \\
\hline & & CTV & Clinical target volume \\
\hline & & PFS & Progression-free survival \\
\hline \multicolumn{2}{|c|}{$\begin{array}{l}\triangle \text { Atsushi Natsume } \\
\text { anatsume@med.nagoya-u.ac.jp }\end{array}$} & CTCAE & $\begin{array}{l}\text { Common terminology criteria for adverse } \\
\text { events }\end{array}$ \\
\hline \multicolumn{2}{|c|}{ Extended author information available on the last page of the article } & ACNU & Nimustine hydrochloride \\
\hline
\end{tabular}


HR Hazard ratio

JNS Japan Neurosurgical Society

IARC International Agency for Research on Cancer

\section{Introduction}

Gliomas account for approximately $40 \%$ of all brain tumors and are thus the most common primary tumors of the central nervous system (CNS) [1]. In particular, glioblastoma $(\mathrm{GBM})$ is one of the most frequent brain tumors in the CNS in adults and is highly malignant, with a median survival time of about 1 year from diagnosis [2]. An international randomized trial by the European Organisation for Research and Treatment of Cancer (EORTC)/National Cancer Institute of Canada that compared concomitant radiotherapy (RT) and temozolomide (TMZ) to RT alone clearly demonstrated the benefits of adjuvant TMZ chemotherapy for GBM patients [3]. The median OS in the GBM patients who received RT + TMZ in trials in Europe [3], the United States [4], and an international collaboration (AVAglio) [5] were 14.6, 16.8, and 15.7 months, respectively.

Since then, TMZ has been the current first-line chemotherapeutic agent for GBM. A subgroup analysis in the trial above revealed the effectiveness of epigenetic silencing of the $O^{6}$-methylguanine-DNA methyltransferase (MGMT) gene via promoter methylation, with longer survival, in patients with primary GBM. It also suggested the benefits of agents targeting $M G M T$ combining with TMZ plus radiotherapy [6]. Interferon $\beta$ (IFN $\beta$ ) exerts pleiotropic biological effects $[7,8]$ and has been widely used either as a single agent or in combination with other antitumor agents in the treatment of malignant gliomas and melanomas [9]. In the treatment of malignant gliomas, IFN $\beta$ can act as a drug sensitizer, and it enhances the toxicity of chemotherapeutic agents against various neoplasms when administered in combination with nitrosourea [10]. Combination therapy with IFN $\beta$ and nitrosourea has been used primarily in the treatment of gliomas in Japan [11]. In our previous in vitro study of human glioma cells, we found that IFN $\beta$ markedly enhanced chemosensitivity to TMZ [12]. This finding suggested that one of the major mechanisms by which IFN $\beta$ enhances chemosensitivity is the downregulation of $M G M T$ transcription via p53 induction. This effect was also observed in an experimental animal model [13]. The results of these 2 studies suggested that chemotherapy with IFN $\beta$ and TMZ with concomitant RT might further improve the clinical outcome of patients with malignant gliomas, comparing to chemotherapy with TMZ alone and concomitant RT. Based on these results, we translated the preclinical evidence to clinical studies. A phase I study showed the safety and feasibility of chemotherapy with IFN $\beta$ and TMZ combined with concomitant radiotherapy $[14,15]$. In addition, a retrospective study demonstrated that addition of IFN $\beta$ for newly diagnosed primary GBM achieved a favorable outcome, particularly in patients with an unmethylated MGMT promoter [16].

Based on the rationale shown above, we conducted a randomized screening phase II trial of chemoradiotherapy with TMZ plus IFN $\beta$ in comparison with chemoradiotherapy with TMZ alone for newly diagnosed GBM (JCOG0911 INTEGRA study), as the Japan Clinical Oncology Group (JCOG) study to explore the superiority of TMZ + IFN $\beta$ therapy to TMZ alone in terms of overall survival (OS) in patients with newly diagnosed GBM.

\section{Materials and methods}

\section{Patients}

For inclusion in the study, patients had to meet all of the following criteria: histologically proven newly diagnosed GBM based upon WHO 2007 (IARC 4th edition); 50\% of the tumor located in supratentorial areas, without involvement of the optic, olfactory nerves, and pituitary gland and without multiple, disseminated, or large tumors in which the planned irradiated target volume exceeds one-third of the whole brain volume; enrollment 3-20 days after surgery; age between 20 and 75 years; Eastern Cooperative Oncology Group (ECOG) performance status of 0-2 or 3 (only if caused by the tumor); no history of previous chemotherapy or radiotherapy; appropriate organ function and written informed consent.

\section{Treatment}

Patients in the TMZ + RT arm received RT (2.0 Gy/fr/day, $30 \mathrm{fr}$ ) with TMZ (75 mg/m ${ }^{2}$, daily) followed by maintenance of TMZ (100-200 mg/m²/day, days $1-5$, every 4 weeks) for 2 years because (1) optimal duration of maintenance temozolomide had not been determined, and (2) the majority of the investigators in this study agreed that the maintenance temozolomide period was $>12$ months.

Patients in the TMZ + IFN $\beta+$ RT arm intravenously received IFN $\beta$ ( $3 \mathrm{MU} /$ body on day 1 , day 3 , and day 5 during RT concomitant period and day 1 , every 4 weeks during the maintenance period) in addition to TMZ + RT (Fig. 1). We determined IFN-beta dosage based on previously published trials, including a Phase I trial [11, 14, 17-20].

RT with concomitant chemotherapy was started within 3 weeks after the surgery. Three-dimensional conformal radiotherapy was planed. Quality assurance reviews were performed at the Radiotherapy Support Center under supervision of JCOG Radiotherapy Committee, with feedback sent to each institution by the RT study coordinator (Minako 


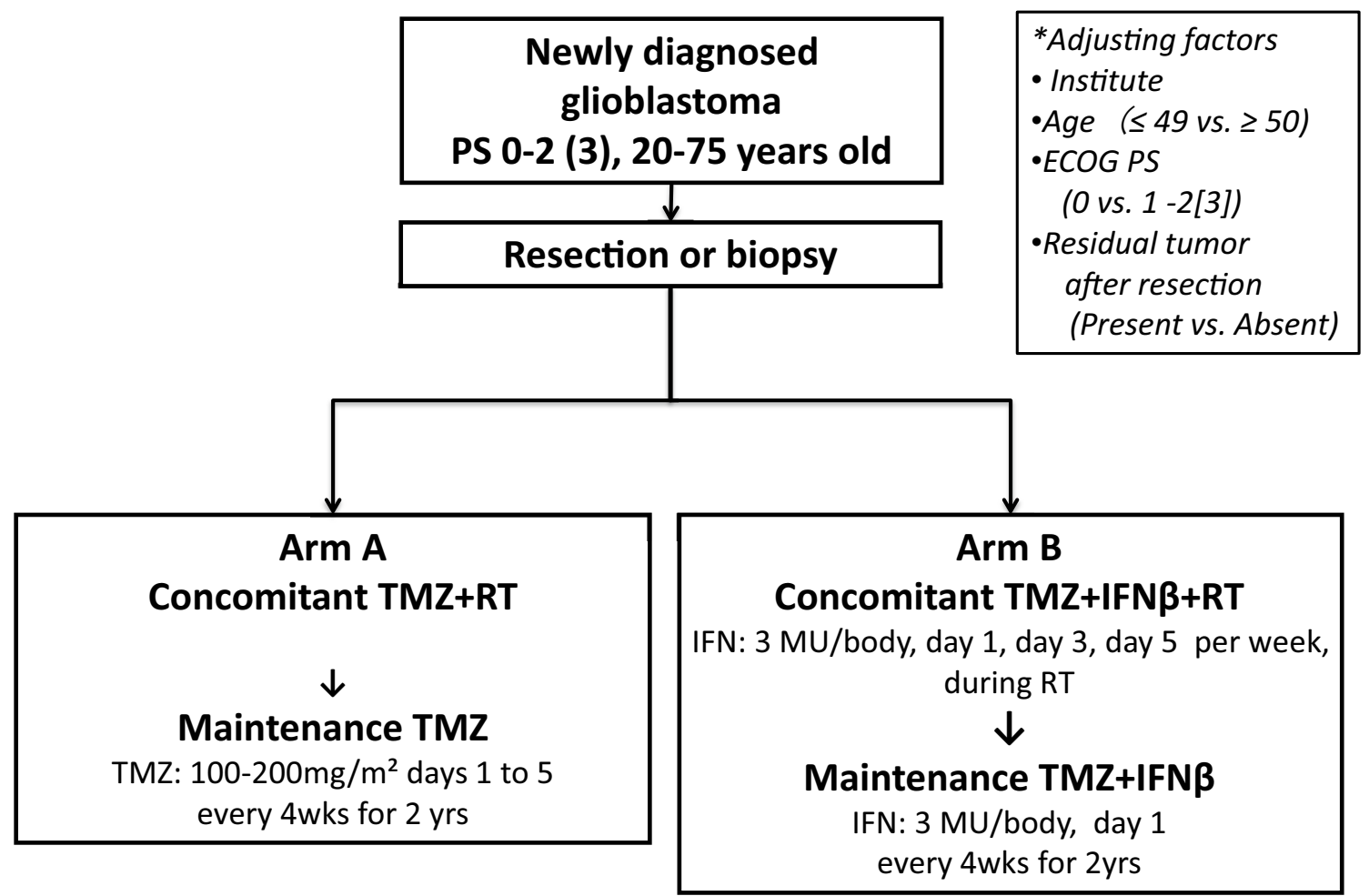

Fig. 1 Patient flow diagram of a randomized screening phase II trial of chemoradiotherapy with interferon $\beta$ plus temozolomide in comparison with chemoradiotherapy with temozolomide alone for newly diagnosed glioblastoma

Sumi). The minimum and maximum doses in the planning target volume (PTV) should comprise between 90 and $110 \%$ of the reference point dose of the International Commission on Radiation Units. Gross tumor volume (GTV) was defined as residual tumor, with or without enhancement on computed tomography or magnetic resonance imaging. The clinical target volume 1 (CTV1) included GTV, the resection cavity, and surrounding edema (high-intensity area on T2-weighted or fluid-attenuated inversion recovery image) plus a $1.5-\mathrm{cm}$ margin. The CTV2 included GTV and the resection cavity plus a $1.5-\mathrm{cm}$ margin. PTV was defined as CTV plus a margin of $0.5 \mathrm{~cm}$. The doses for PTV1 and PTV2 were 50 Gy in 25 fractions and 10 Gy in 5 fractions, respectively.

\section{Study design}

This trial was designed as a multicenter, prospective, randomized screening phase II study to explore the superiority of TMZ + IFN $\beta$ therapy to TMZ alone in terms of OS in patients with newly diagnosed GBM and to decide whether $\mathrm{TMZ}+\mathrm{IFN} \beta$ should be evaluated in a succeeding confirmatory phase III trial. Patients were randomized using a minimization method with biased-coin assignment to receive either the standard arm (TMZ + RT) or the experimental $\operatorname{arm}(\mathrm{TMZ}+\mathrm{IFN} \beta+\mathrm{RT})$ at the JCOG Data Center, adjusting for factors including institution, age ( $\leq 49$ vs. $\geq 50$ years), ECOG performance status ( 0 vs. 1 or 2 [3 if this was due to brain tumor]), and residual tumor after resection (present vs. absent). The study protocol was approved by the JCOG Protocol Review Committee and the institutional review board of each participating institution, and carried out in accordance with the Declaration of Helsinki. This trial was registered at the UMIN Clinical Trials Registry as UMIN000003466 (http://www.umin.ac.jp/ctr/index.htm).

\section{Statistical consideration}

The primary endpoint was OS. OS was calculated from the date of randomization until death from any cause. The secondary endpoints were progression-free survival (PFS), complete response rate, overall response rate, and adverse events. PFS was calculated from the date of randomization until the date of documented progression or death. Responses were evaluated according to Response Evaluation Criteria in Solid Tumors version 1.0. Toxicities were evaluated according to the Common Terminology Criteria for Adverse Events (CTCAE) version 3.0.

The planned sample size was 120 and the expected number of events was 70, with a one-sided alpha of 0.2 and power of 0.8 to detect a difference between arms. The 1-year survival was presumed to be $65 \%$ in the $\mathrm{TMZ}+\mathrm{RT}$ arm, and 
was expected to be $75 \%$ in the TMZ + IFN $\beta+\mathrm{RT}$ arm. The planned accrual and follow-up period were 1.5 and 2 years, respectively. Primary analysis was conducted 2 year after the accrual completion.

One interim analysis was scheduled after the half of the planned sample size was enrolled to assess the futility of this study. Multiplicity was not taken into consideration because terminating the trial due to superiority of TMZ $+\mathrm{IFN} \beta+\mathrm{RT}$ arm was not planned. Results of interim analysis were reviewed by the JCOG Data and Safety Monitoring Committee and investigators were masked to the results.

OS was analyzed by the stratified log-rank test with residual tumor after resection (present vs. absent) as a strata. Hazard ratio was estimated by stratified Cox proportional hazard model with residual tumor after resection (present vs. absent) as a strata. PFS was analyzed by the unstratified logrank test and unstratified Cox proportional hazard model. OS and PFS curves were estimated by the Kaplan-Meier method. The efficacy analyses were by intention-to treat and safety analyses were by all patients who received protocol treatment. All analyses were performed by the JCOG Data Center using SAS 9.2 (SAS Institute, Cary, NC).

\section{Results}

\section{CONSORT diagram and characteristics of the ITT population}

From April 2010 to January 2012, 122 patients were accrued, of whom 63 and 59 patients were assigned to the $\mathrm{TMZ}+\mathrm{RT}$ and $\mathrm{TMZ}+\mathrm{IFN} \beta+\mathrm{RT}$ arms, respectively (Fig. 2). All the tumors were proven to be GBM by the central pathological review. In addition, IDH1/2 mutation in each tumor was not detected though anti-IDH1-R132H immunohistochemistry and Sanger sequencing (Table 1). The patients' characteristics were as follows: median age (61 years [range $22-75$ years] vs. 61 years [range $30-73$ years]), male/female (38/25 vs. $35 / 24)$, ECOG performance status $0 / 1-3$ (16/47 vs. 12/47), residual tumor resection absent/present (31/32 vs. 33/26) (Table 1). One patient in the TMZ + IFN $\beta+$ RT arm was off-protocol before the initiation of protocol treatment owing to liver

Table 1 Characteristics of the ITT population

\begin{tabular}{lll}
\hline & TMZ+RT $(\mathrm{n}=63)$ & $\begin{array}{l}\text { TMZ+ IFN } \beta+\mathrm{RT} \\
(\mathrm{n}=59)\end{array}$ \\
\hline $\begin{array}{l}\text { Age, median (range) } \\
\text { Gender }\end{array}$ & $61(22-75)$ & $61(30-73)$ \\
Male & 38 & 35 \\
Female & 25 & 24 \\
ECOG PS & & \\
0 & 16 & 12 \\
$1-3$ & 47 & 47 \\
Residual tumor after resection & \\
Absent & 31 & 33 \\
Present & 32 & 26 \\
IDH1/2 status & & \\
No mutation & 57 & 58 \\
Not examined & 6 & 1 \\
\hline
\end{tabular}

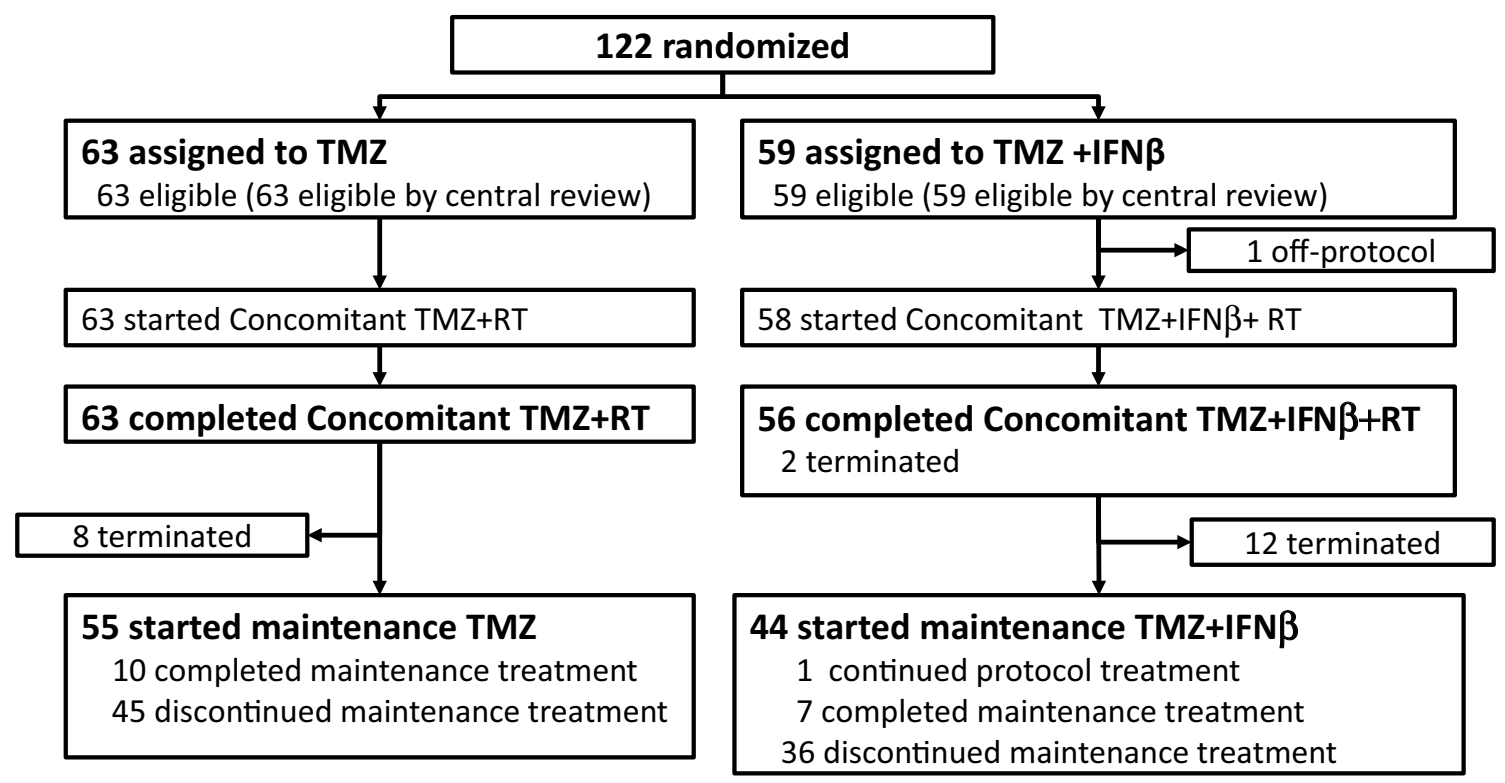

Fig. 2 Consort diagram. From April 2010 to January 2012, 122 patients were accrued, of whom 63 and 59 patients were assigned to the $\mathrm{TMZ}+\mathrm{RT}$ and TMZ + IFN $\beta+\mathrm{RT}$ arms, respectively 
Table 2 Number of courses of the maintenance treatments

\begin{tabular}{lclc}
\hline $\begin{array}{l}\text { Number of } \\
\text { treatment } \\
\text { course }\end{array}$ & TMZ+RT $(\mathrm{n}=63)$ & $\begin{array}{l}\text { TMZ+IFN } \beta+R T \\
(\mathrm{n}=56)\end{array}$ & $\mathrm{n}=119$ \\
\hline 0 & $8(12.7 \%)$ & $12(21.4 \%)$ & 20 \\
$1-12$ & $39(61.9 \%)$ & $29(51.8 \%)$ & 68 \\
$13-31$ & $16(25.4 \%)$ & $15(26.8 \%)$ & 31 \\
\hline
\end{tabular}

Table 3 Post-protocol treatments

\begin{tabular}{lcl}
\hline & $\begin{array}{l}\text { RT/TMZ } \\
(\mathrm{n}=39)\end{array}$ & $\begin{array}{l}\text { RT/TMZ/ } \\
\text { IFN } \beta \\
(\mathrm{n}=39)\end{array}$ \\
\hline 1. Same as protocol treatment & 11 & 14 \\
TMZ & 9 & 5 \\
TMZ+ IFN $\beta$ & 2 & 9 \\
2. Other chemotherapy (ACNU, Irinote- & 1 & 6 \\
can, ICE, other TMZ regimens) & & \\
3.SRS, SRT & 4 & 7 \\
4. Surgery & 18 & 11 \\
5. Others & 5 & 1 \\
Bevacizumab & 3 & 0 \\
Vaccine & 2 & 1 \\
\hline
\end{tabular}

dysfunction and thus was excluded from the safety analysis. Two patients in the TMZ + IFN $\beta+$ RT arm terminated protocol treatment during the concomitant period due to progression and adverse events (grade 3 anorexia and grade 2 erythema multiforme) and 12 patients terminated protocol treatment due to progression, adverse events and patient refusal with adverse events (7, 3, and 2 patients) in the interval between the concomitant and the maintenance treatments. In the TMZ + RT arm, 8 patients terminated protocol treatment during the interval between the concomitant and the maintenance treatments because of progression or adverse events ( 7 and 1 patients). In the maintenance period, 55 patients started TMZ, but 45 patients terminated the maintenance treatment owing to progression, adverse events, and patient refusal with adverse events $(34,3$, and 6 patients) in the TMZ + RT arm. In the $\mathrm{TMZ}+\mathrm{IFN} \beta+\mathrm{RT}, 44$ patients started TMZ + IFN $\beta$, but 36 patients terminated the maintenance treatment owing to progression, adverse events, patient refusal with adverse events, and another reason $(30,1,3$, and 1 patients) (Table 2). One treatment related death (TRD) was observed in TMZ + IFN $\beta+\mathrm{RT}$ arm during the maintenance therapy (severe renal failure). The post-protocol treatments are listed in Table 3. Chemotherapy using either TMZ or $\mathrm{TMZ}+\mathrm{IFN} \beta$ was administered as post-protocol treatments in 11 and 14 patients, respectively. Other chemotherapies were applied in 1 and 6 patients. Bevacizumab was used in 3 patients in the TMZ + RT arm.

\section{Overall and progression-free survival}

The median survival time was 20.3 months (95\% CI 15.4-26.9 months) and 24.0 months (95\% CI 18.8-27.4 months) in the TMZ + RT arm and the TMZ + IFN $\beta+$ RT arm, respectively (HR 1.00, 95\% CI 0.65-1.55; one-sided $\log$ rank $\mathrm{P}=0.51)$. OS did not statistically differ between the two arms (Fig. 3a).

The median PFS was 10.1 months (95\% CI $7.5-11.8$ months) and 8.5 months (95\% CI 6.6-11.9 months) in the $\mathrm{TMZ}+\mathrm{RT}$ arm and the TMZ + IFN $\beta+\mathrm{RT}$ arm, respectively (HR 1.25, 95\% CI 0.85-1.84; two-sided $\mathrm{P}=0.25$ ) (Fig. 3b).

Subgroup analyses were performed for OS by sex (male/ female), age ( $\leq 49$ years $>50$ years), residual tumor after resection (absent/present) and ECOG PS (0/1/2-3) (Fig. 4). Male, Younger patients ( $\leq 49$ years) and ECOG PS 0 in the $\mathrm{TMZ}+\mathrm{IFN} \beta+\mathrm{RT}$ arm showed good OS compared with RT/ TMZ arm.

\section{Adverse events}

The incidence of grade 3 and 4 neutropenia was higher in the TMZ + IFN $\beta+$ RT arm (Table 4). The difference was more marked in the patients aged $\geq 50$ years. Among the non-hematological adverse events, fever, nausea/vomiting, and appetite loss tended to be more frequent in the $\mathrm{TMZ}+\mathrm{IFN} \beta+\mathrm{RT}$ arm.

\section{Discussion}

IFNs exert pleiotropic antitumor effects by direct anticancer mechanisms though p53 induction and miR-21 downregulation or by regulating the immune system through the CD8 lymphocyte and macrophage activation [7]. This study was a randomized screening phase II trial to explore the superiority of TMZ + IFN $\beta$ therapy to TMZ alone for the patients with newly diagnosed GBM. In the present study, the superiority of $\mathrm{TMZ}+\mathrm{IFN} \beta+\mathrm{RT}$ to $\mathrm{TMZ}+\mathrm{RT}$ in $\mathrm{OS}$ was not demonstrated.

There are some possibilities that we failed to show the superiority of TMZ + IFN $\beta+R T$ to $T M Z+R T$. One potential reason is that TMZ + IFN $\beta+R T$ treatment was more toxic than expected. Before we started this trial, we assumed that additional IFN $\beta$ would not increase much toxicity because it had been suggested in some reports using nitrosourea anti-tumor agent with IFN $\beta$. However, the proportion of severe (grade 3-4) hematological and non-hematological adverse events was higher in the $\mathrm{TMZ}+\mathrm{IFN} \beta+\mathrm{RT}$ arm than in the TMZ + RT arm, which 
a

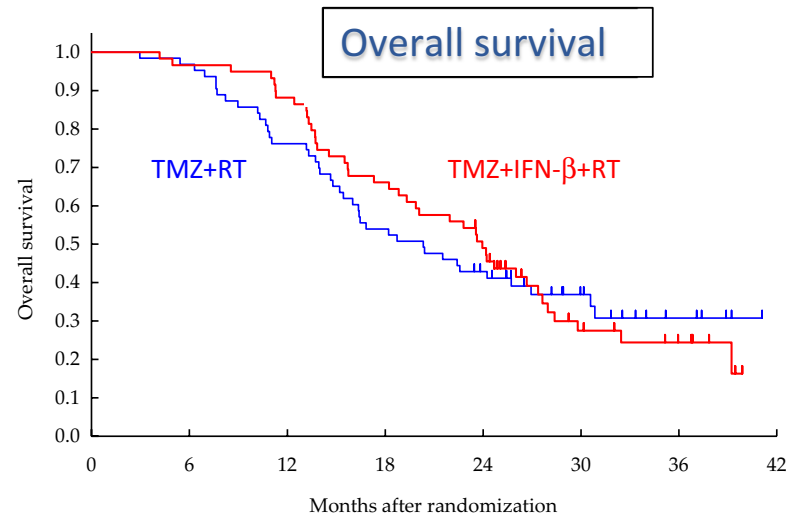

\begin{tabular}{|l|c|c|}
\hline & $\begin{array}{c}\text { TMZ+RT } \\
\mathrm{N}=63\end{array}$ & $\begin{array}{c}\text { TMZ+IFN- } \beta+\mathrm{RT} \\
\mathrm{N}=59\end{array}$ \\
\hline $\begin{array}{l}\text { MST } \\
(95 \% \mathrm{Cl})\end{array}$ & $\begin{array}{c}20.3 \mathrm{~m} \\
(15.4 \mathrm{~m}-26.9 \mathrm{~m})\end{array}$ & $\begin{array}{c}24.0 \mathrm{~m} \\
(18.8 \mathrm{~m}-27.4 \mathrm{~m})\end{array}$ \\
\hline Stratified HR $(95 \% \mathrm{Cl})$ & \multicolumn{2}{|c|}{$1.00(0.65-1.55)$} \\
\hline $\begin{array}{l}\text { One-sided p-value } \\
\text { by stratified log-rank test }\end{array}$ & \multicolumn{2}{|c|}{$\mathrm{P}=0.51>0.20=\alpha$} \\
\hline
\end{tabular}

Fig. 3 Clinical outcomes. The median survival time was 20.3 months (95\% CI 15.4-26.9 months) and 24.0 months (95\% CI 18.8-27.4 months) in the TMZ+RT arm and the TMZ+ IFN $\beta+$ RT arm, respectively (HR 1.00, 95\% CI $0.65-1.55$; one-sided log rank $\mathrm{P}=0.51)$. OS did not statistically differ between the two arms (a).

b

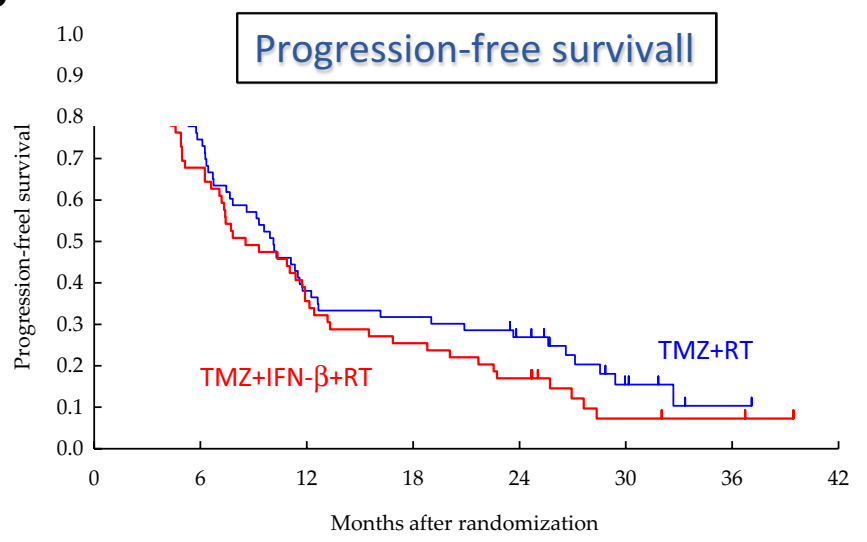

\begin{tabular}{|l|c|c|}
\hline & $\begin{array}{c}\text { TMZ+RT } \\
\mathrm{N}=63\end{array}$ & $\begin{array}{c}\text { TMZ+IFN- } \beta+R T \\
\mathrm{~N}=59\end{array}$ \\
\hline MST $(95 \% \mathrm{Cl})$ & $\begin{array}{c}10.1 \mathrm{~m} \\
(7.5 \mathrm{~m}-11.8 \mathrm{~m})\end{array}$ & $\begin{array}{c}8.5 \mathrm{~m} \\
(6.6 \mathrm{~m}-11.9 \mathrm{~m})\end{array}$ \\
\hline Unstratified HR $(95 \% \mathrm{Cl})$ & \multicolumn{2}{|c|}{$1.25(0.85-1.84)$} \\
\hline $\begin{array}{l}\text { Two-sided } p \text {-value } \\
\text { by unstratified log-rank test }\end{array}$ & \multicolumn{2}{|c|}{$\mathrm{P}=0.25$} \\
\hline
\end{tabular}

The median PFS was 10.1 months (95\% CI 7.5-11.8 months) and 8.5 months (95\% CI 6.6-11.9 months) in the TMZ + RT arm and the TMZ+IFN $\beta+$ RT arm, respectively (HR 1.25, 95\% CI 0.85-1.84; two-sided $\mathrm{P}=0.25)(\mathbf{b})$

Subgroup analysis of OS

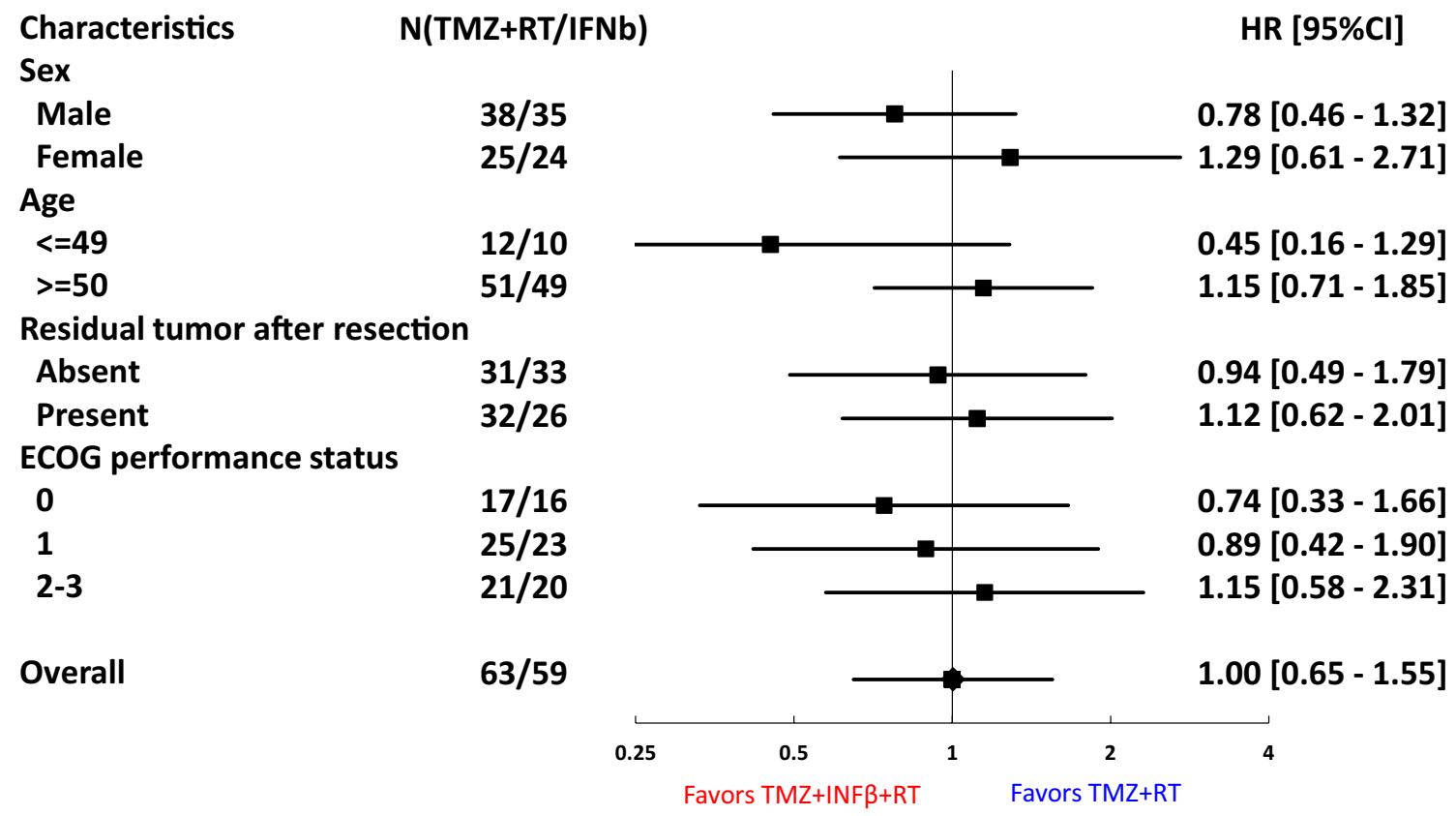

Fig. 4 Subgroup analyses were performed for OS by sex (male/ female), age ( $\leq 49$ years $>50$ years), residual tumor after resection (absent/present) and ECOG PS (0/1/2-3). Male, Younger patients
( $\leq 49$ years) and ECOG PS 0 in the TMZ + IFN $\beta+$ RT arm showed good OS compared with RT/TMZ arm 
Table 4 (a) Adverse events (concomitant chemoradiotherapy), (b) adverse events (maintenance therapy)

\begin{tabular}{|c|c|c|c|c|c|c|}
\hline \multirow[t]{2}{*}{ (a) } & \multicolumn{3}{|c|}{$\mathrm{TMZ}+\mathrm{RT}(\mathrm{N}=63)$} & \multicolumn{3}{|c|}{$\mathrm{TMZ}+\mathrm{IFN} \beta+\mathrm{RT}(\mathrm{N}=58)$} \\
\hline & Grade $1-2(\%)$ & Grade $3(\%)$ & Grade $4(\%)$ & Grade $1-2(\%)$ & Grade $3(\%)$ & Grade $4(\%)$ \\
\hline \multicolumn{7}{|l|}{ Hematological } \\
\hline Neutropenia & $25(39.7)$ & $4(6.3)$ & $4(6.3)$ & $32(55.2)$ & $10(17.2)$ & $2(3.5)$ \\
\hline Lymphopenia & $24(38.1)$ & $28(44.4)$ & $6(9.5)$ & $20(34.5)$ & $30(51.7)$ & $7(12.1)$ \\
\hline \multicolumn{7}{|l|}{ Non-hematological } \\
\hline Fever & $12(19.0)$ & 0 & 0 & $18(31.0)$ & $1(1.7)$ & 0 \\
\hline Nausea & $18(28.6)$ & 0 & 0 & $18(31.0)$ & $2(3.4)$ & 0 \\
\hline Vomiting & $7(11.1)$ & 0 & 0 & $10(17.2)$ & $1(1.7)$ & 0 \\
\hline Anorexia & $26(41.3)$ & 0 & 0 & $26(44.8)$ & $5(8.6)$ & 0 \\
\hline Febrile neutropenia & - & $1(1.6)$ & 0 & - & $2(3.4)$ & 0 \\
\hline ALT elevation & $35(55.6)$ & $6(9.5)$ & 0 & $31(53.4)$ & $5(8.6)$ & $1(1.7)$ \\
\hline Hyponatremia & $13(20.6)$ & $3(4.8)$ & 0 & $15(25.9)$ & $5(8.6)$ & 0 \\
\hline Skin rash & $13(20.6)$ & $1(1.6)$ & 0 & $4(6.9)$ & 0 & 0 \\
\hline \multirow[t]{2}{*}{ (b) } & \multicolumn{3}{|c|}{$\mathrm{TMZ}+\mathrm{RT}(\mathrm{N}=55)$} & \multicolumn{3}{|c|}{$\mathrm{TMZ}+\mathrm{IFN} \beta+\mathrm{RT}(\mathrm{N}=43)^{\mathrm{a}}$} \\
\hline & Grade $1-2(\%)$ & Grade $3(\%)$ & Grade $4(\%)$ & Grade 1-2(\%) & Grade $3(\%)$ & Grade $4(\%)$ \\
\hline \multicolumn{7}{|l|}{ Hematological } \\
\hline Neutropenia & $31(56.4)$ & $2(3.6)$ & 0 & $21(48.8)$ & $4(9.3)$ & 0 \\
\hline Lymphopenia & $29(52.7)$ & $17(30.9)$ & $2(3.6)$ & $22(51.2)$ & $17(39.5)$ & $1(2.3)$ \\
\hline \multicolumn{7}{|l|}{ Non-hematological } \\
\hline Fever & $3(5.5)$ & 0 & 0 & $5(11.6)$ & 0 & 0 \\
\hline Nausea & $14(25.5)$ & 0 & 0 & $6(14.0)$ & 0 & 0 \\
\hline Vomiting & $4(7.3)$ & $1(1.8)$ & 0 & $2(4.7)$ & 0 & 0 \\
\hline Anorexia & $16(29.1)$ & $2(3.6)$ & 0 & $7(16.3)$ & 0 & 0 \\
\hline Febrile neutropenia & - & 0 & 0 & - & 0 & 0 \\
\hline ALT elevation & $30(54.5)$ & $1(1.8)$ & 0 & $20(46.5)$ & 0 & 0 \\
\hline Hyponatremia & $11(20.0)$ & 0 & 0 & $7(16.3)$ & $2(4.7)$ & 0 \\
\hline Skin rash & $8(14.5)$ & 0 & 0 & 8 (18.6) & 0 & 0 \\
\hline
\end{tabular}

According to the Common Terminology Criteria for Adverse Events (CTCAE) version 3.0

${ }^{a}$ Data of one patient is missing

implied such unexpected severe toxicities could cause negative impact on the survival in the TMZ + IFN $\beta+$ RT arm. Due to the severe toxicities, treatment compliance was also deteriorated in the TMZ + IFN $\beta+\mathrm{RT}$ arm. In fact, the number of patients who terminated protocol treatment before the start of the maintenance treatments was larger in the TMZ + IFN $\beta+$ RT arm, which was possibly caused by the toxicities as mentioned above. MGMT methylation status has not been investigated yet, but we are planning to evaluate the biomarkers including $M G M T$ gene expression and methylation using tumor tissues and blood samples.

Subgroup analyses showed IFN $\beta$ could be possibly beneficial for younger, male, better PS, no residual tumor patients. It may suggest that the better tolerability against IFN $\beta$ toxicities might be predictive factors of IFN $\beta$ efficacy, but further studies would be needed to confirm this hypothesis.
As the future direction, we will seek for the promising combination therapy with $\mathrm{TMZ}+\mathrm{RT}$ and other agents than IFN $\beta$ as a candidate of the following study for GBM. Now we just started a randomized phase III trial to confirm the superiority of dose-dense TMZ (ddTMZ) followed by bevacizumab at ddTMZ failure to bevacizumab alone for patients with first recurrence or progression of GBM (JCOG1308C).

In conclusion, although the combination therapy of $\mathrm{TMZ}+\mathrm{IFN} \beta+\mathrm{RT}$ showed favorable survival, the superiority of TMZ/IFN $\beta+\mathrm{RT}$ to $\mathrm{TMZ}+\mathrm{RT}$ in overall survival was not demonstrated. Therefore TMZ + IFN $\beta+$ RT was not considered promising as the test treatment in the following phase III study for newly diagnosed GBM and TMZ+ RT remained to be a most promising treatment.

Acknowledgements We thank all the members of the JCOG Brain Tumor Study Group and the staff of the JCOG Data Center. This work 
was supported in part of the National Cancer Center Research and Development Fund (20S-4, 23-A-20, 26-A-4, 29-A-3), the Health and Labour Science Research Grant (H20-19), and the Grant-in Aid for Cancer Research (H23-013) from the ministry of Health, Labour and Welfare, Japan.

\section{Compliance with ethical standards}

Conflict of interest T.W. has received research funding from Toray Co, Ltd, and MSD Co. Ltd. K.S. received honorarium fundings from MSD. M.N. received honorarium and research fundings from MSD, Chugai, and Eisai, and research funding from Daiichi-Sankyo. R.N. received honorarium and research fundings from MSD, Chugai, and Eisai. The other authors declare that they have no conflict of interest. All authors have registered online Self-reported COI Disclosure Statement Forms through the website for Japan Neurosurgical Society members.

Open Access This article is distributed under the terms of the Creative Commons Attribution 4.0 International License (http://creativeco mmons.org/licenses/by/4.0/), which permits unrestricted use, distribution, and reproduction in any medium, provided you give appropriate credit to the original author(s) and the source, provide a link to the Creative Commons license, and indicate if changes were made.

\section{References}

1. Ostrom QT, Gittleman H, Liao P, Vecchione-Koval T, Wolinsky Y, Kruchko C, Barnholtz-Sloan JS (2017) CBTRUS statistical report: primary brain and other central nervous system tumors diagnosed in the United States in 2010-2014. Neuro Oncol 19:v1-v88. https://doi.org/10.1093/neuonc/nox158

2. Stupp R, Hegi ME, Mason WP, van den Bent MJ, Taphoorn MJ, Janzer RC, Ludwin SK, Allgeier A, Fisher B, Belanger K, Hau P, Brandes AA, Gijtenbeek J, Marosi C, Vecht CJ, Mokhtari K, Wesseling P, Villa S, Eisenhauer E, Gorlia T, Weller M, Lacombe D, Cairncross JG, Mirimanoff RO, European Organisation for Research and Treatment of Cancer Brain Tumour and Radiation Oncology Groups, National Cancer Institute of Canada Clinical Trials Group (2009) Effects of radiotherapy with concomitant and adjuvant temozolomide versus radiotherapy alone on survival in glioblastoma in a randomised phase III study: 5-year analysis of the EORTC-NCIC trial. Lancet Oncol 10:459-466 https://doi.org/10.1016/S1470-2045(09)70025-7

3. Stupp R, Mason WP, van den Bent MJ, Weller M, Fisher B, Taphoorn MJ, Belanger K, Brandes AA, Marosi C, Bogdahn U, Curschmann J, Janzer RC, Ludwin SK, Gorlia T, Allgeier A, Lacombe D, Cairncross JG, Eisenhauer E, Mirimanoff RO, European Organisation for Research and Treatment of Cancer Brain Tumor and Radiotherapy Groups, National Cancer Institute of Canada Clinical Trials Group (2005) Radiotherapy plus concomitant and adjuvant temozolomide for glioblastoma. $\mathrm{N}$ Engl J Med 352: 987-996 https://doi.org/10.1056/NEJMoa0433 30

4. Gilbert MR, Dignam JJ, Armstrong TS, Wefel JS, Blumenthal DT, Vogelbaum MA, Colman H, Chakravarti A, Pugh S, Won M, Jeraj R, Brown PD, Jaeckle KA, Schiff D, Stieber VW, Brachman DG, Werner-Wasik M, Tremont-Lukats IW, Sulman EP, Aldape KD, Curran WJ Jr, Mehta MP (2014) A randomized trial of bevacizumab for newly diagnosed glioblastoma. N Engl J Med 370:699-708. https://doi.org/10.1056/NEJMoa1308573

5. Chinot OL, Wick W, Mason W, Henriksson R, Saran F, Nishikawa R, Carpentier AF, Hoang-Xuan K, Kavan P, Cernea D,
Brandes AA, Hilton M, Abrey L, Cloughesy T (2014) Bevacizumab plus radiotherapy-temozolomide for newly diagnosed glioblastoma. N Engl J Med 370:709-722. https://doi. org/10.1056/NEJMoa1308345

6. Hegi ME, Diserens AC, Gorlia T, Hamou MF, de Tribolet N, Weller M, Kros JM, Hainfellner JA, Mason W, Mariani L, Bromberg JE, Hau P, Mirimanoff RO, Cairncross JG, Janzer RC, Stupp R (2005) MGMT gene silencing and benefit from temozolomide in glioblastoma. N Engl J Med 352:997-1003

7. Galani V, Papadatos SS, Alexiou G, Galani A, Kyritsis AP (2017) In vitro and in vivo preclinical effects of type I IFNs on gliomas. J Interferon Cytokine Res 37:139-146. https://doi. org/10.1089/jir.2016.0094

8. GuhaSarkar D, Su Q, Gao G, Sena-Esteves M (2016) Systemic AAV9-IFNbeta gene delivery treats highly invasive glioblastoma. Neuro Oncol 18:1508-1518. https://doi.org/10.1093/ neuonc/now097

9. Chawla-Sarkar M, Lindner DJ, Liu YF, Williams BR, Sen GC, Silverman RH, Borden EC (2003) Apoptosis and interferons: role of interferon-stimulated genes as mediators of apoptosis. Apoptosis 8:237-249

10. Kawaji H, Tokuyama T, Yamasaki T, Amano S, Sakai N, Namba $\mathrm{H}$ (2015) Interferon-beta and temozolomide combination therapy for temozolomide monotherapy-refractory malignant gliomas. Mol Clin Oncol 3:909-913. https://doi.org/10.3892/ mco.2015.542

11. Wakabayashi T, Hatano N, Kajita Y, Yoshida T, Mizuno M, Taniguchi K, Ohno T, Nagasaka T, Yoshida J (2000) Initial and maintenance combination treatment with interferon-beta, MCNU (Ranimustine), and radiotherapy for patients with previously untreated malignant glioma. J Neurooncol 49:57-62

12. Natsume A, Ishii D, Wakabayashi T, Tsuno T, Hatano H, Mizuno M, Yoshida J (2005) IFN-beta down-regulates the expression of DNA repair gene MGMT and sensitizes resistant glioma cells to temozolomide. Cancer Res 65:7573-7579. https://doi. org/10.1158/0008-5472.CAN-05-0036

13. Natsume A, Wakabayashi T, Ishii D, Maruta H, Fujii M, Shimato S, Ito M, Yoshida J (2007) A combination of IFN-beta and temozolomide in human glioma xenograft models: implication of p53-mediated MGMT downregulation. Cancer Chemother Pharmacol 61:653-659

14. Wakabayashi T, Kayama T, Nishikawa R, Takahashi H, Hashimoto N, Takahashi J, Aoki T, Sugiyama K, Ogura M, Natsume A, Yoshida J (2011) A multicenter phase I trial of combination therapy with interferon-beta and temozolomide for highgrade gliomas (INTEGRA study): the final report. J Neurooncol 104:573-577. https://doi.org/10.1007/s11060-011-0529-1

15. Wakabayashi $\mathrm{T}$, Kayama $\mathrm{T}$, Nishikawa R, Takahashi $\mathrm{H}$, Yoshimine T, Hashimoto N, Aoki T, Kurisu K, Natsume A, Ogura M, Yoshida J (2008) A multicenter phase I trial of interferon-beta and temozolomide combination therapy for highgrade gliomas (INTEGRA study). Jpn J Clin Oncol 38:715-718. https://doi.org/10.1093/jjco/hyn095

16. Motomura K, Natsume A, Kishida Y, Higashi H, Kondo Y, Nakasu Y, Abe T, Namba H, Wakai K, Wakabayashi T (2011) Benefits of interferon-beta and temozolomide combination therapy for newly diagnosed primary glioblastoma with the unmethylated MGMT promoter: a multicenter study. Cancer 117:1721-1730. https://doi.org/10.1002/cncr.25637

17. Yoshida J, Kajita Y, Wakabayashi T, Sugita K (1994) Long-term follow-up results of 175 patients with malignant glioma: importance of radical tumour resection and postoperative adjuvant therapy with interferon, ACNU and radiation. Acta Neurochir 127:55-59

18. Hatano N, Wakabayashi T, Kajita Y, Mizuno M, Ohno T, Nakayashiki N, Takemura A, Yoshida J (2000) Efficacy of post 
operative adjuvant therapy with human interferon beta, MCNU and radiation (IMR) for malignant glioma: comparison among three protocols. Acta Neurochir 142:633-638 (discussion 639)

19. Watanabe T, Katayama Y, Yoshino A, Fukaya C, Yamamoto T (2005) Human interferon beta, nimustine hydrochloride, and radiation therapy in the treatment of newly diagnosed malignant astrocytomas. J Neurooncol 72:57-62. https://doi.org/10.1007/ s11060-004-2160-x
20. Colman H, Berkey BA, Maor MH, Groves MD, Schultz CJ, Vermeulen S, Nelson DF, Mehta MP, Yung WK, Radiation Therapy Oncology G (2006) Phase II Radiation Therapy Oncology Group trial of conventional radiation therapy followed by treatment with recombinant interferon-beta for supratentorial glioblastoma: results of RTOG 9710. Int J Radiat Oncol Biol Phys 66:818-824. https://doi.org/10.1016/j.ijrobp.2006.05.021

\section{Affiliations}

Toshihiko Wakabayashi ${ }^{1} \cdot$ Atsushi Natsume $^{1}$ (D) Junki Mizusawa ${ }^{2} \cdot$ Hiroshi Katayama $^{2} \cdot$ Haruhiko Fukuda $^{2}$. Minako Sumi ${ }^{3} \cdot$ Ryo Nishikawa $^{4} \cdot$ Yoshitaka Narita $^{5} \cdot$ Yoshihiro Muragaki $^{6} \cdot$ Takashi Maruyama $^{6}$. Tamio Ito $^{7}$. Takaaki Beppu $^{8} \cdot$ Hideo Nakamura $^{9} \cdot$ Takamasa Kayama $^{10}$. Shinya Sato ${ }^{10} \cdot$ Motoo Nagane $^{11} \cdot$ Kazuhiko Mishima $^{4}$. Yoko Nakasu ${ }^{12} \cdot$ Kaoru Kurisu $^{13} \cdot$ Fumiyuki Yamasaki $^{13} \cdot$ Kazuhiko Sugiyama $^{14} \cdot$ Takanori Onishi $^{15} \cdot$ Yasuo Iwadate $^{16}$. Mizuhiko Terasaki ${ }^{17}$. Hiroyuki Kobayashi ${ }^{18}$. Akira Matsumura ${ }^{19}$. Eiichi Ishikawaa ${ }^{19}$. Hikaru Sasaki ${ }^{20}$.

Akitake Mukasa ${ }^{21}$. Takayuki Matsuo ${ }^{22}$. Hirofumi Hirano ${ }^{23} \cdot$ Toshihiro Kumabe $^{24,25}$. Nobusada Shinoura ${ }^{26}$. Naoya Hashimoto ${ }^{27}$. Tomokazu Aoki ${ }^{28}$. Akio Asai ${ }^{29} \cdot$ Tatsuya Abe $^{30}$. Atsuo Yoshino ${ }^{31}$. Yoshiki Arakawa ${ }^{32}$. Kenichiro Asano ${ }^{33} \cdot$ Koji Yoshimoto $^{34}$. Soichiro Shibui ${ }^{5}$. Members of Japan Clinical Oncology Group Brain Tumor Study Group (JCOG-BTSG)

1 Department of Neurosurgery, Nagoya University Graduate School of Medicine, Nagoya, Japan

2 JCOG (Japan Clinical Oncology Group) Data Center/Operations Office, National Cancer Center Hospital, Tokyo, Japan

3 Radiation Oncology Department, Cancer Institute Hospital, Tokyo, Japan

4 Department of Neuro-Oncology/Neurosurgery, Saitama Medical University International Medical Center, Saitama, Japan

5 Department of Neurosurgery and Neuro-Oncology, National Cancer Center Hospital, Tokyo, Japan

6 Department of Neurosurgery, Tokyo Women's Medical University, Tokyo, Japan

7 Department of Neurosurgery, Nakamura Memorial Hospital, Sapporo, Japan

8 Department of Neurosurgery, Iwate Medical University, Morioka, Japan

9 Department of Neurosurgery, Kumamoto University Graduate School of Medicine, Kumamoto, Japan

10 Department of Neurosurgery, Yamagata University Graduate School of Medicine, Yamagata, Japan

11 Department of Neurosurgery, Kyorin University Faculty of Medicine, Mitaka, Japan

12 Department of Neurosurgery, Shizuoka Cancer Center, Shizuoka, Japan

13 Department of Neurosurgery, Hiroshima University, Graduate School of Biomedical and Health Sciences, Hiroshima, Japan

14 Department of Clinical Oncology \& Neuro-oncology Program, Hiroshima University Hospital, Hiroshima, Japan

15 Department of Neurosurgery, Ehime University Graduate School of Medicine, Ehime, Japan
16 Department of Neurosurgery, Chiba University Graduate School of Medicine, Chiba, Japan

17 Department of Neurosurgery, Kurume University Graduate School of Medicine, Kurume, Japan

18 Department of Neurosurgery, Hokkaido University Graduate School of Medicine, Sapporo, Japan

19 Department of Neurosurgery, Faculty of Medicine, University of Tsukuba, Tsukuba, Japan

20 Department of Neurosurgery, Keio University School of Medicine, Tokyo, Japan

21 Department of Neurosurgery, The University of Tokyo Hospital, Tokyo, Japan

22 Department of Neurosurgery, Nagasaki University Graduate School of Medicine, Nagasaki, Japan

23 Department of Neurosurgery, Kagoshima University Graduate School of Medical and Dental Sciences, Kagoshima, Japan

24 Department of Neurosurgery, Tohoku University Graduate School of Medicine, Sendai, Japan

25 Present Address: Department of Neurosurgery, Kitasato University School of Medicine, Kanagawa, Japan

26 Department of Neurosurgery, Tokyo Metropolitan Cancer and Infectious Disease Center Komagome Hospital, Tokyo, Japan

27 Department of Neurosurgery, Osaka University Graduate School of Medicine, Suita, Japan

28 Department of Neurosurgery, Kitano Hospital, Osaka, Japan

29 Department of Neurosurgery, Kansai Medical University, Osaka, Japan

30 Department of Neurosurgery, Oita University Faculty of Medicine, Yufu, Japan

31 Department of Neurological Surgery, Nihon University Graduate School of Medicine, Tokyo, Japan 
32 Department of Neurosurgery, Kyoto University Graduate School of Medicine, Kyoto, Japan

33 Department of Neurosurgery, Hirosaki University Graduate School of Medicine, Hirosaki, Japan
34

Department of Neurosurgery, Graduate School of Medical Sciences, Kyushu University, Fukuoka, Japan 